\title{
Nanoparticle-Mediated Cytoplasmic Delivery of Messenger RNA Vaccines: Challenges and Future Perspectives
}

\author{
Zimei Wu' (1) • Tonglei Li
}

Received: 14 January 2021 / Accepted: 10 February 2021 / Published online: 3 March 2021

(C) The Author(s), under exclusive licence to Springer Science+Business Media, LLC part of Springer Nature 2021

\begin{abstract}
The COVID-19 pandemic has left scientists and clinicians no choice but a race to find solutions to save lives while controlling the rapid spreading. Messenger RNA (mRNA)-based vaccines have become the front-runners because of their safety profiles, precise and reproducible immune response with more cost-effective and faster production than other types of vaccines. However, the physicochemical properties of naked mRNA necessitate innovative delivery technologies to ferry these 'messengers' to ribosomes inside cells by crossing various barriers and subsequently induce an immune response. Intracellular delivery followed by endosomal escape represents the key strategies for cytoplasmic delivery of mRNA vaccines to the target. This Perspective provides insights into how state-of-the-art nanotechnology helps break the delivery barriers and advance the development of mRNA vaccines. The challenges remaining and future perspectives are outlined.
\end{abstract}

KEY WORDS endosomal escape · intracellular delivery . $m R N A$ vaccines $\cdot$ nanomedicines - ribosome

\section{INTRODUCTION}

The year 2020 began with a grave situation as the world was engulfed by the novel severe acute respiratory syndrome coronavirus 2 (SARS-GoV-2), which causes coronavirus disease-

Zimei Wu

z.wu@auckland.ac.nz

School of Pharmacy, Faculty of Medical and Health Sciences, The University of Auckland, Auckland I I42, New Zealand

2 Department of Industrial and Physical Pharmacy, College of Pharmacy, Purdue University, 575 Stadium Mall Drive, West

Lafayette, Indiana 47907, USA
2019 (COVID-19). In response, scientists and clinicians around the world raced up to a search for therapeutics as well as prophylactic vaccines to stop the rapid spreading. With an unprecedented speed, the end of 2020 saw the front-runners, two nanomedicine-based messenger RNA (mRNA) vaccines BNT162b2 and mRNA-1273 received Emergency Use Authorization (EUA) from the US Food and Drug Administration (FDA), as the first-ever mRNA medicines. Several potential mRNA vaccines are also in the pipeline (Table I). Apart from the tremendous efforts in SARS-CoV2 genome sequencing (1), such a fast pace of progress could not have been possible without the rapid identifications of antigens and an mRNA sequence, and the preparedness in drug delivery sciences (2). The cutting-edge drug delivery technologies played a pivotal role in fully realizing the potential of the mRNA to elicit a robust immune response (3). Besides, the long-term experience in good manufacturing practice (GMP) and scale-up abilities of the (bio) pharmaceutical industry must have been instrumental in accelerating the vaccine programs during clinical translation. This Perspective here will briefly explain the underlying mechanisms of this type of vaccines and elucidate how drug delivery sciences help mRNA cross the physiological barriers to their target. We will primarily focus on lipid-based nano-platforms. Furthermore, the challenges remaining, and future research required will be considered.

\section{Advantages of mRNA Vaccines}

The mRNA vaccine is a newly emerged gene-based technology following its first report in the 1990s when an mRNA was injected into animals and produced measurable encoded proteins (8). Since then, mRNA has attracted significant attention not only as vaccine platforms against infectious diseases and cancer but also as protein replacement therapies for many diseases (9). As for vaccines, either as conventional nonreplicating or self-amplifying mRNA (saRNA), mRNA 
Table I Examples of Lipid Nanoparticle (LNP) Empowered Messenger RNA (mRNA) Vaccines to Combat COVID-19 at Various Stages of Development*. All are Administered Intramuscularly (IM) as Two Injections in 3-4 Weeks Apart

\begin{tabular}{|c|c|c|c|}
\hline Vaccine name & Developer(s) & Formulation/Reference & Stage of development \\
\hline BNTI $62 \mathrm{~b} 2$ & $\begin{array}{l}\text { BioNTech / Fosun } \\
\text { Pharma / Pfizer }\end{array}$ & LNP-mRNAs (4) & FDA EUA \\
\hline mRNA- 1273 & Moderna / NIAID ${ }^{\mathrm{a}}$ & LNP-encapsulated mRNA (5) & FDA EUA \\
\hline CVnCoV mRNA & CureVac & LNP-mRNA & $\begin{array}{l}\text { Phase Illb/lll } \\
\text { NCT } 04652102\end{array}$ \\
\hline ARCT-02I & $\begin{array}{l}\text { Arcturus Therapeutics / Duke-NUS } \\
\text { Medical School }\end{array}$ & $\begin{array}{l}\text { LUNARß }(\mathrm{pH} \text {-sensitive LNP-mediated } \\
\text { delivery of saRNA) }\end{array}$ & $\begin{array}{l}\text { Phase II } \\
\text { NCT04480957 } \\
\text { NCT04668339 }\end{array}$ \\
\hline LNP-nCoVsaRNA & Imperial College London & $\begin{array}{l}\text { LNP-saRNA (proprietary, cationic, } \\
\text { PEGylated) (6) }\end{array}$ & $\begin{array}{l}\text { Phase I } \\
\text { ISRCTN I } 7072692\end{array}$ \\
\hline ARCoV & $\begin{array}{l}\text { PLAAMS } / \\
\text { Walvax Biotech }\end{array}$ & LNP-mRNA (7) & $\begin{array}{l}\text { Phase I } \\
\text { ChiCTR2000034 I I 2; } \\
\text { ChiCTR20000392 I } 2\end{array}$ \\
\hline
\end{tabular}

*A few other types of COVID vaccines, including denatured virus Vero cell, and non-replicating viral vectors AZD I 222 (Oxford-AstraZeneca) and Ad26.COV2.S (Johnson \& Johnson) have also progressed to Phase III clinical trials (clinical trial number ChiCTR2000034780, NCT045 I 6746 and NCT04505722 respectively)

${ }^{a}$ NIAID: National Institute of Allergy and Infectious Diseases; ' ${ }^{2}$ PLAAMS: People's Liberation Army Academy of Military Sciences; saRNA: self-amplifying (or replicating) mRNA. ${ }^{c}$ Arcturus Therapeutics website (https://ir.arcturusrx.com/press-releases)

vaccines contain a single-stranded genetic sequence instructing the host cells to generate proteins within ribosomes to yield immune responses, thus priming the immune system against pathogens or cancer cells. For example, the COVID-19 mRNA vaccines currently developed are encoding for spike glycoprotein (S protein), which is expressed on the surface of SARS-CoV-2. The virus uses $\mathrm{S}$ protein to gain entry into the alveolar cells of lungs via the specific receptor, angiotensinconverting enzyme-II (ACE2) (1). Therefore, S protein is a key target to combat COVID-19 (6). Compared with other vaccine types (inactivated, viral vectors, protein subunit or DNA-based), mRNA vaccines possess a number of advantages, including (10, 11): 1) Safe and reliable transfection. mRNAs do not integrate into the genome of host cells but only are transient carriers of information for explicit protein synthesis. Therefore, mRNA vaccines are not considered genetic-modified materials in contrast to DNA vaccines; Repeat administration may be required to achieve adequate immune response (Table I). In this regard, saRNA can induce equivalent protection effects at a significantly lower dose than the conventional mRNAs, and possibly with a single dose (6). 2) Cost-effective, rapid and scalable production. As mRNAs have well-defined chemical structures (sequence), state-of-theart biotechnology makes it possible for large-scale synthesis via in vitro transcribed (IVT) from a DNA template in a cell-free system. This also means a lesser stringent safety requirement and quality control, compared with the traditional virus-based vaccine production. 3) Reliable induction of both $\mathrm{T}$ cell (cellular) and B cell (humoral) immune responses, giving a double strike at the virus. Briefly, the expressed proteins (EPs) released from the host cells are captured by the antigen-presenting cells (APCs), primarily dendritic cells
(DCs). The EPs are subsequently degraded to antigenic peptides within the cells and presented to cytotoxic $\mathrm{T}$ lymphocytes via the major histocompatibility complex (MHC) I pathway, and to B-cells via the MHC II pathway (12). Therefore, targeting mRNA directly to APCs, mainly DCs, is the most effective approach to immunization. Alternatively, mRNA vaccines may indirectly transfect bystander cells (host cells), such as myocytes.

\section{NANOPARTICLES MEDIATED CYTOPLASMIC DELIVERY OF MRNA}

Despite the aforementioned advantages, safe and efficient delivery of mRNA to the ribosomes in the cytoplasm of target cells is a major obstacle to clinical translation (8). Due to their large molecular size, hydrophilicity, negative charge, and fragile nature, naked mRNA can barely cross the lipophilic cell membranes (also slightly negatively charged) by diffusion. Instead, they are internalized via caveolae/lipid raft-rich membrane domains, presumably with the involvement of scavenger-receptors (13). However, following endocytosis, mRNAs eventually get accumulated in lysosomes where they are subsequently degraded, with only a small proportion being released into cytosol (13) (Fig. 1). In addition, like any nucleic acid, mRNAs are intrinsically unstable and prone to degradation by the extracellular ribonucleases (RNases) before internalized. Thanks to the advances in nanoscience, a wide range of nano-carriers have been constructed in recent years to facilitate cytoplasmic delivery of mRNA (14). The nanoplatforms that have been exploited for in vivo delivery of mRNA include lipid nanoparticles (LNPs; including cationic 


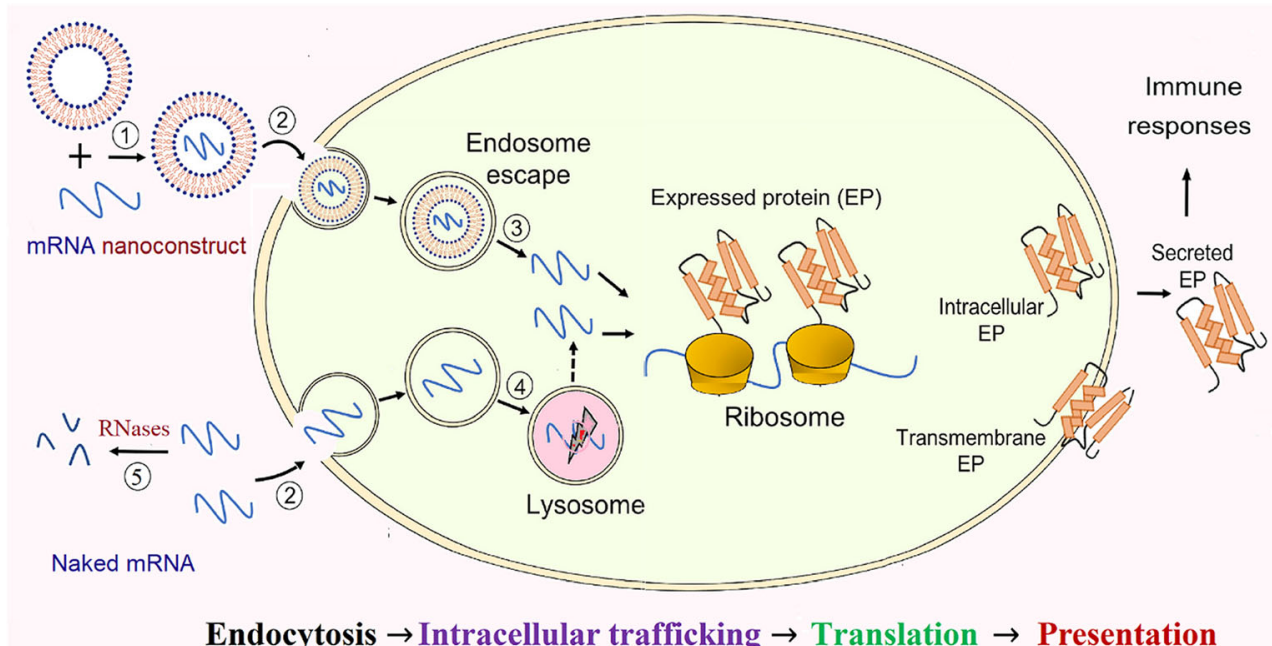

Fig. I Simplified schematic representation of cellular uptake and intracellular trafficking of naked mRNA versus $L N P$ encapsulated mRNA. I) mRNA is loaded into various nanoparticles, 2) mRNA nanoconstruct or naked mRNA is internalized via different endocytosis pathways (clathrin- and caveolae-mediated, or lipid raft raft), 3) mRNA nanoconstruct undergoes endosomal escape leading to cargo release into cytosol and ribosome, 4) naked mRNA ended in lysosomes and subsequently degraded with only a small proportion escaped into the cytosol, and 5) extracellular unprotected mRNA undergoes enzymatic degradation.

liposomes/lipoplexes with lipid bilayers, and more often with a lipid monolayer and lipophilic cores), polymer-based nanoparticles, lipid-polymer hybrids (15-17). Among these nonviral vectors, LNPs are particularly appealing and have been widely used as mRNA carriers (8), because of their biocompatible properties, ability to protect the mRNA against degradation, and flexibility to be tailored to target specific cell types (APCs, such as DCs) by surface modification with a ligand $(16,17)$. Importantly, tremendous progress in manufacturing and scale-up technologies have also made the implementation of LNP-based mRNA medicines possible, evidenced by the mRNA vaccines that have already been progressed to clinical trials for cancer treatment (17) as well as the recent success in COVID-19 vaccines as shown in Table I.

\section{Formulation Compositions}

A rationally designed mRNA delivery system can fulfill at least four roles: promote mRNA entrapment efficiency, protect the mRNA from degradation, enable cellular uptake, and facilitate endosomal escape (Fig. 1). The performance is dominated by the formulation components and physicochemical properties of the nanostructures as well as the biological factors. As mRNAs are negatively charged owing to the presence of phosphate groups in their backbone, mRNA carrier systems are typically composed of a cationic lipid or polymer to allow ionization at lower $\mathrm{pH}$ to facilitate encapsulation and/or complexation with an mRNA. These lipids or polymers usually bear tertiary amine groups with a $p \mathrm{Ka}$ slightly lower than 7.0, which allows them to be largely neutral at the physiological $\mathrm{pH}$. In addition, a typical LNP system also contains cholesterol, $\mathrm{pH}$-sensitive lipids (helper lipids), and a poly(ethylene glycol) (PEG)-lipid (stealth lipid), which contribute to stabilization of the LNP membrane, promoting endosomal escape, and reducing opsonization by serum proteins in vivo, respectively (18). Numerous ionizable cationic lipids and polymers (some are proprietary, such as MC3) have been developed, as summarized in the literature $(8,14,19)$. It is worth noting that 1,2-dioleoyl-3trimethylammonium propane (DOTAP, $p \mathrm{Ka} 6.3$ ) is one of the most widely used cationic lipids in mRNA and gene delivery with proved in vitro and in vivo transfection efficiency. The zwitterionic phospholipid, 1,2-dioleoylphosphatidylethanolamine (DOPE) has been one of the mostly used helper lipids, owing to its additional fusogenicity that favors both cellular uptake and intracellular trafficking.

LNP-mRNAs can be assembled using the ethanol injection precipitation method in which the lipid components dissolved in an ethanolic solution is rapidly mixed with mRNA in an acidic aqueous solution $(\mathrm{pH} 4-5)(2,6)$. The low $\mathrm{pH}$ is crucial in yielding high encapsulation efficiencies for mRNA by ensuring protonation of the cationic lipids. The PEG-lipid could either be added in the ethanoic phase (2) (pre-insertion) or post-inserted to the preformed LNP-mRNA. Physiological $\mathrm{pH}$ of the final product can be achieved by dialysis of the LPN against an aqueous buffer. Based on this principle, the recent advances in microfluidic mixing devices have allowed fast and large-scale manufacture of LNP-mRNA. Other technique such as emulsion method, (ethanol is replaced with an oil phase) usually with aid of homogenization can also be used to prepare LNP-mRNA. 


\section{Routes of Administration and Pharmacokinetics}

The routes of administration need to be considered as part of formulation design and are critical for different therapeutic applications of mRNAs (14). mRNA vaccines have been administered via various parenteral routes in the preclinical studies, including intravenous (IV), intradermal (ID), subcutaneous (SC), and intramuscular (IM) injections $(8,14)$. The route of administration dominates the pharmacokinetics (tissue distribution and clearance), the intensity of local/systemic reactions (both inflammatory or therapeutic), and ultimately the immune responses (20). Each of the routes offers different advantages and requires different formulation strategies to overcome their limitations. When mRNA is directly delivered to APCs, translation and antigen presentation could be realized in the same cells, thus eliciting a maximal immune response. It has been recorded that the effect of cancer mRNA vaccines was correlated with the number of transfected DCs (16).

The LNP-mRNA vaccines for COVID-19 are IM administered (Table I). After IM injection, the myocytes take up the LNPs effectively prior to the cytoplasmic release of the mRNAs for translation of S protein (5). This route permits a relatively larger volume to be injected than the ID and SC routes and thus may induce less adverse injection site reactions, albeit more systemic absorption (22). Depending on their local spreadability, diffusivity, release rate, and tissue permeability, IM injected LNP-mRNA and their encoded antigen proteins can presumably reach the DCs that are populated in the SC lymph nodes as well as the APCs in the muscle. From this aspect, mRNA vaccines in lipid-based nanoconstructs are advantageous as they tend to be transported to lymphatic systems in which APCs are in close proximity to T cells (21). However, limited information is reported about the local transport and absorption kinetics.

Some data demonstrated that IM, as well as ID delivery of LNP-mRNA vaccines, resulted in more persistent protein expression than IV routes (8). ID injection of the vaccine favorably allows exposure to Langerhans cells, a specialized subset of DCs populated in the epidermis layer of the skin. In contrast, the SC route offers the possibility to directly activate the DCs that are residing in the lymph nodes near the injection site. However, injection volume for either SC or ID is limited compared to IM injection.

\section{Cellular Uptake and Endosomal Escape}

Cellular uptake is conceivably the primary limiting-step determining the bioavailability of mRNA to the host cells. Apart from the selection of lipids or polymers, manipulation of particle size and surface charge can also enhance the internalization efficiency. However, successful intracellular delivery will not necessarily result in RNA translation. Nanoparticles are internalized mainly via clathrin- and caveolae-mediated endocytosis, which leads to subsequent endosome entrapment, limiting the cellular bioavailability of mRNA (Fig. 1). Therefore, endosomal escape is another key step to deliver mRNA to ribosomes, and there is a strong correlation between endosomal escape and transfection efficiency (23).

In general, nanoparticles undergo endosomal escape by three hypothetical pathways: i) dissociation/destabilization under acidic lumen of endosome; ii) fusion of liposomes with endosomal membrane, and iii) rupture of endosomal membranes. Different nanoconstructs exploit different pathway(s): for example, 'pH-responsive endosomal escape' and 'proton sponge effect'. 1) pH-responsive conformational changes due to protonation, or cleavage of a bond in the polymer at endosomes lead to 'pH-responsive endosomal escape' (18). Cationic lipids may interact with the negatively charged endosomal membrane to facilitate endosomal escape (14). DOPE becomes fusogenic following the protonation of its head group at acidic conditions, leading to the formation of a hexagonal $\left(\mathrm{H}_{\mathrm{II}}\right)$ phase and temporarily destabilizing the endosomal membrane; 2) Cationic polyplexes are considered to undergo endosomal escape through the 'proton sponge effect', where endocytosed polyplexes induce an osmotic swelling of the endosome due to the influx of protons and eventually cause the endosome to rupture (14).

\section{CHALLENGES AND OUTLOOK}

Overall, enhanced intracellular delivery followed by endosomal escape has proven to be successful delivery strategies in the recent approval of mRNA vaccines. Yet, there remain some key questions, thus opportunities to reinforce the translocation efficiency of mRNA, targetability, and patient compliance. In addition, research is also is urgently needed to address the thermal stability of mRNA vaccines during the storage and transportation.

\section{Translocation Efficiency and Transfection}

Fully understanding the intracellular trafficking of the mRNA-nanosystems and the influencing factors will provide insights into the development of more effective and safer delivery strategies. However, factors such as nitrogen: phosphate groups $(\mathrm{N}: \mathrm{P})$ ratios, particle size and surface charge, and their interplays with cytoplasmic delivery efficiencies have not been well understood. Notably, research in small interfering RNA (siRNA) delivery suggests that only less than $2 \%$ of the administered LNPs-siRNA escapes from the endosome entrapment reaching the cytosol, as summarized by the Valadi group (24). This indicates that there is still a large room to improve translocation efficiency via endosomal escape pathways. Besides, it is unknown whether it is the naked mRNA alone or the LNP- 
mRNA escaped from endosomes (16). It is envisaged that at the acidic lumens of endosome and lysosomes ( $\mathrm{pH} 5-6)$ favors the complexation of cationic lipids with the negatively charged mRNA, rather than releasing mRNA.

Interestingly, it was recently reported that endocytosed LNP-mRNA was re-packaged into extracellular vesicles (EV) and secreted from the recipient cells (24). This 'exocytosis' phenomenon points to an alternative direction to the production of nano-carriers (EV) to deliver mRNAs. It would also be of interest to research whether this phenomenon can be utilized to promote cell-to-cell transfection via the biological functions of $\mathrm{EV}$ in cell-to-cell communication.

\section{Cell Targetability}

More specific APC-targeting would lead to increased transfection efficiency alongside reduced off-target side effects by two ways: selective delivery of mRNA to DCs and promoted uptake via receptor-ligand mediated endocytosis. Of note, mRNA-'targeting' is intended for maximal amount of administered mRNA-nanoparticle to be targeted to a sufficient number of DCs, rather than targeting a large quantities of nanoparticles to the same DCis.

To enhance the specific cellular uptake by APGs, selection of a proper route of delivery is important, as discussed above. With each administration route, formulation parameters for optimization may vary including lipid composition, particle size, surface (net) charge, the degree of PEGylation, and the selection of targeting ligands based on the location of targeted tissues.

Surface modification with a specific ligand would result in more specific, and rapid uptake via the receptor-mediated mechanisms. For example, mannosylated-PEG-lipids have been widely investigated for small molecule drug delivery and may be exploited for targeting APCs since both DGs and macrophages reportedly carry sugar receptors (16).

\section{PEG Dilemma and Anaphylactic Reactions}

PEGylation brings the "PEG dilemma" - steric stabilization in vitro and protection of rapid clearance in viwo versus hindrance of both cellular uptake and endosomal escape (18). In addition, PEG polymers may trigger the production of anti-PEG antibodies, causing the accelerated blood clearance (ABC) phenomenon in the subsequent dosing. Therefore, the reported LNP formulations for mRNA delivery have rarely used PEG2000-lipid more than $1.5 \mathrm{~mol} \%$.

PEGylated vaccines may elicit life threatening anaphylactic reactions in people previously have high levels of anti-PEG antibodies. Severe anaphylactic reactions have been reported following administration of the mRNA vaccines (26). The US Centers for Disease Control and Prevention (CDC) has urged that individuals who are allergic to PEG (and polysorbate) should not get an mRNA COVID-19 vaccine and others should be observed for 15 min after injection (26).

Therefore, PEG alternatives with no antigenicity may be an imminent next step in research. It is well known that hydrophilic polymers, such as poly(N-(2-hydroxypropyl)-methacrylamide) (HPMA) and polyaminoacids, can be used to confer stealth properties to nanoparticles without causing immunogenicity. Another question remains unanswered is whether PEG dilemma can be ameliorated by de-PEGylation strategies by which PEG is cleaved off within the host cells by intracellular stimuli (25).

In terms of delivery platforms, endogenous nano-sized EVs (30-150 nm, also known as exosomes) secreted from cells have emerged as a new delivery vehicle with distinct advantages, including biocompatibility, non-immunogenicity, tissue penetration, and cell-tropism with products under clinical trials (27). As per review (8), ex vivo packing autologous DCs with mRNA (electroporation) has been practised to achieve precise control of the cellular target. Intriguingly, this loading process can be adopted to generate mRNA-loaded EVs in vitro for delivery purposes potentially for saRNAs. However, scale-up production poses challenging.

\section{Patient Compliance and Precision Medicine}

Research is needed to overcome the injection site reactions associated with ID, SC, and IM administration (edema and erythema), which are likely to be caused by the ionizable components (22). Furthermore, depending on the stability of the mRNA, sustained release technologies in formulation design to reduce the dosing times from two doses to a single injection is highly desirable. With distinguished advantages over parenteral administration to improve patient compliance, the non-invasive intranasal and pulmonary mRNA vaccines may offer an attractive option for combating respiratory infectious diseases. Through rational formulation designs, these routes may target mucosal immune cells by overcoming the mucosal barrier in addition to the cellular barriers to evoke potent immunity in the respiratory system (28).

More importantly, understanding and eventually predicting local and systemic transport and absorption kinetics of a delivery system, which can be further coupled with downstream pharmacological and immune responses, are urgently needed. Such quantitative systems pharmacology approaches establish the connection among formulation design, patient conditions, and treatment outcome, ensuring the safety and maximizing the efficacy.

\section{CONCLUSION}

In summary, the global COVID-19 pandemic has motivated the successful development of safe, potent, and scalable 
mRNA vaccines. It opens an exciting era in mRNA medicines not only for vaccination but also for therapeutics to tackle many unmet medical needs - a 'silver lining' of this deadly disease. Cytoplasmic delivery strategies with nanoconstructs have primarily circumvented the major barriers to ferry mRNAs to their target. Future work is needed to shed more light on further understanding of formulation variables on cellular uptake, intracellular trafficking mechanisms, and thus harnessing the full potential of mRNA medicines with formulation design. New nanomaterials to strengthen endosomal escape ability, and solve the 'PEG dilemma' may be of great importance to improve the efficacy and safety. Therefore, multidisciplinary collaborative research efforts are required from biologists, formulation and biomaterial scientists, and clinicians.

\section{REFERENCES}

1. Zhou P, Yang X-L, Wang X-G, Hu B, Zhang L, Zhang W, et al. A pneumonia outbreak associated with a new coronavirus of probable bat origin. Nature. 2020;579(7798):270-3 .

2. Corbett KS, Edwards DK, Leist SR, Abiona OM, BoyogluBarnum S, Gillespie RA, et al. SARS-CoV-2 mRNA vaccine design enabled by prototype pathogen preparedness. Nature. 2020;586(7830):567-71.

3. Weiss C, Carriere M, Fusco L, Capua I, Regla-Nava JA, Pasquali M. Toward nanotechnology-enabled approaches against the COVID-19 pandemic. ACS Nano. 2020;14(6):6383-406.

4. Polack FP, Thomas SJ, Kitchin N, Absalon J, Gurtman A, Lockhart S, et al. Safety and efficacy of the BNT162b2 mRNA Covid-19 vaccine. N Engl J Med. 2020;383(27):2603-15.

5. Wang F, Kream RM, Stefano GB. An evidence based perspective on mRNA-SARS-GoV-2 vaccine development. Med Sci Monit. 2020;26:e924700.

6. McKay PF, Hu K, Blakney AK, Samnuan K, Brown JC, Penn R, et al. Self-amplifying RNA SARS-GoV-2 lipid nanoparticle vaccine candidate induces high neutralizing antibody titers in mice. Nat Commun. 2020;11(1):3523.

7. Zhang N-N, Li X-F, Deng Y-Q Zhao H, Huang Y-J, Yang G, et al. A thermostable mRNA vaccine against COVID-19. Cell. 2020;182(5):1271-83.e16.

8. Pardi N, Hogan MJ, Porter FW, Weissman D. mRNA vaccines - a new era in vaccinology. Nat Rev Drug Discov. 2018;17(4):261-79.

9. Wadhwa A, Aljabbari A, Lokras A, Foged C, Thakur A. Opportunities and challenges in the delivery of mRNA-based vaccines. Pharmaceutics. 2020;12(2).

10. Krammer F. SARS-CoV-2 vaccines in development. Nature. 2020;586(7830):516-27.

11. Tombácz I, Weissman D, Pardi N. Vaccination with messenger RNA: a promising alternative to DNA vaccination. Methods Mol Biol. 2021;2197:13-31.
12. Xu S, Yang K, Li R, Zhang L. mRNA vaccine era-mechanisms, drug platform and clinical prospection. Int J Mol Sci. 2020;21(18):6582.

13. Lorenz G, Fotin-Mleczek M, Roth G, Becker G, Dam TC, Verdurmen WP, et al. Protein expression from exogenous mRNA: uptake by receptor-mediated endocytosis and trafficking via the lysosomal pathway. RNA Biol. 201 1;8(4):627-36.

14. Li B, Zhang X, Dong Y. Nanoscale platforms for messenger RNA delivery. Wiley interdiscip Rev Nanomed Nanobiotechnol. 2019;11(2):e1530.

15. Guevara ML, Persano F, Persano S. Advances in lipid nanoparticles for mRNA-based Cancer immunotherapy. Front Chem. 2020;8:589959.

16. Midoux P, Pichon C. Lipid-based mRNA vaccine delivery systems. Expert Rev Vaccines. 2015;14(2):221-34.

17. Reichmuth AM, Oberli MA, Jaklenec A, Langer R, Blankschtein D. mRNA vaccine delivery using lipid nanoparticles. Ther Deliv. 2016;7(5):319-34.

18. Kanamala M, Wilson WR, Yang M, Palmer BD, Wu Z. Mechanisms and biomaterials in pH-responsive tumour targeted drug delivery: a review. Biomaterials. 2016;85:152-67.

19. Kauffman KJ, Webber MJ, Anderson DG. Materials for non-viral intracellular delivery of messenger RNA therapeutics. J Control Release. 2016;240:227-34.

20. Pardi N, Tuyishime S, Muramatsu H, Kariko K, Mui BL, Tam YK, et al. Expression kinetics of nucleoside-modified mRNA delivered in lipid nanoparticles to mice by various routes. J Control Release. 2015;217:345-51.

21. Kranz LM, Diken M, Haas H, Kreiter S, Loquai C, Reuter KC, et al. Systemic RNA delivery to dendritic cells exploits antiviral defence for cancer immunotherapy. Nature. 2016;534(7607):396401.

22. Hassett KJ, Benenato KE, Jacquinet E, Lee A, Woods A, Yuzhakov $\mathrm{O}$, et al. Optimization of lipid nanoparticles for intramuscular administration of mRNA vaccines. Mol Ther Nucleic Acids. 2019;15: $1-11$.

23. Jiang Y, Lu Q, Wang Y, Xu E, Ho A, Singh P, et al. Quantitating Endosomal escape of a library of polymers for mRNA delivery. Nano Lett. 2020;20(2):1117-23.

24. Maugeri M, Nawaz M, Papadimitriou A, Angerfors A, Camponeschi A, Na M, et al. Linkage between endosomal escape of LNP-mRNA and loading into EVs for transport to other cells. Nat Commun. 2019;10(1):4333.

25. Kanamala M, Palmer BD, Jamieson SM, Wilson WR, Wu Z. Dual $\mathrm{pH}$-sensitive liposomes with low $\mathrm{pH}$-triggered sheddable PEG for enhanced tumor-targeted drug delivery. Nanomedicine (Lond). 2019;14(15):1971-89.

26. Banerji A, Wickner PG, Saff R, Stone CA Jr, Robinson LB, Long $\mathrm{AA}$, et al. mRNA vaccines to prevent COVID-19 disease and reported allergic reactions: current evidence and suggested approach. J Allergy Clin Immunol Pract. 2020;S2213-2198(20): 31411-2.

27. Geng T, Pan P, Leung E, Chen Q, Chamley L, Wu Z. Recent advancement and technical challenges in developing small extracellular vesicles for cancer drug delivery. Pharm Res.In Press.

28. Jia Y, Krishnan L, Omri A. Nasal and pulmonary vaccine delivery using particulate carriers. Expert Opin Drug Deliv. 2015;12(6): 993-1008.

Publisher's Note Springer Nature remains neutral with regard to jurisdictional claims in published maps and institutional affliliations. 